CYBERNETICS AND INFORMATION TECHNOLOGIES • Volume 15, No 6 Special Issue on Logistics, Informatics and Service Science

\title{
An Heuristic Order Promising Method Based on Short-Term Production Capacity Balancing Planning
}

\section{Z. Lv, T. Li, J. Zhang}

University of Science \& Technology Beijing, National Engineering Research Centre for Advanced Rolling, Beijing, China

Emails: lvzhimin@nercar.ustb.edu.cn_ctdpgy@hotmail.com_fmandjay@163.com

Abstract: In the MTO (Make-To-Order) and MTS (Make-To-Stock) mixed production environment, it is important to make an accurate delivery promise on the ordering stage because the customer's demand with unmatched production capacity is not always satisfied. A quick order promising method for an iron and steel enterprise, based on short-term production capacity balancing planning is proposed in this paper. With the help of this method, ATP (Available-To-Promise) concept is extended to RATP (Resource-Available-To-Promise) and DTP (DeliveryTo-Promise) is given while making the resource promise. A heuristic method is developed to match the available resources with the order demand and the actual data testing results have shown that the method proposed can meet the demand of online order promising in a relatively short time.

Keywords: Order promising, short-term production capacity balancing plan, resource Available-To-Promising (ATP), Delivery Date-To-Promising (DTP), steel plant.

\section{Introduction}

With the development of steel and iron market, the enterprises are facing the problem that the market demands have gradually converted to great varieties, small batch and on time delivery. Steel makers are now enthusiastic about increasing their service level to attract more potential customers [11]. In China, a mixed MTO (Make-To-Order) and MTS (Make-To-stock) production mode has been adopted by many steel and iron enterprises for satisfying the special demands and accurate delivery promising. Meanwhile, these enterprises make timely adjustment of the MTS products to respond to the new orders [13]. Therefore, a reasonable order promising system is necessarily needed for the purpose of improving the customer service level and guaranteeing the production balance. Order promising, which is 
the prerequisite and basis of the production operation plan in Advanced Planning System (APS), makes the preliminary commitment of the quantity and delivery time for received customer's inquiry orders and is divided into two modules: ATP (Available-To-Promise) and CTP (Capacity-To-Promise) [4]. ATP adopts stock matching to meet the customers' orders while the surplus capacity of the companies is utilized by the delivery time promising in CTP.

Order allocation and promising have become major concern problems recently [9]. Lu Shan [7] studied a system framework on a supply chain-oriented multistage ATP. Ho Sang Jung [6] formulated an ATP model based on customer's priority and variance of the penalty costs. Deterministic linear programming models for ATP allocation and ATP consumption are established by Her b e r t M e y r [8]. Juin-Han Chen proposed a two-phase order promising process, in which ATP is first reserved in phase I and customer orders are promised in phase II (see [3]).

In the MTO and MTS mixed production environment, ATP and CTP conditions are not always satisfied. Christoph Hempsch embedded multi-attribute simulations into a linear program to fulfill the customer order [5]. B u i and S ebastian [1] considered ATP and CTP functions in a production-distribution network and presented a hybrid and iterative method, based on a MILP model. It is essential to make efficient customer promising and reduce the inventory, in order to promote the cooperation of production planning and sales commitments [2].

In summary, the researches on order promising mainly focus on employing stock matching and available capacity promising to the customers' orders in steel enterprises, though capacity has always dynamic and uncertain characteristics [12]. However, other resources like alternative resources and surplus production capacity have been ignored. Moreover, because of the particularity of iron and steel production in MTS and MTO coexistence production mode, cooperating production planning and sale planning should be taken into account for achieving customer promising and resource balance.

According to the characteristics of MTO and MTS mixed production mode of iron and steel enterprises, in this paper ATP concept is expanded into overall resource matching which is MTS planning; alternative resources, inventory, capacity resources and all available resources promising are considered RATP (Resource-Available-To-Promise) [10]. For obtaining accurate promising of the customer delivery time in the ordering stage and offering personalized management for special requirements of customers in the whole process, the concept of Delivery-To-Promise (DTP) was proposed.

\section{Problem description and model formulation}

\subsection{Problem description}

On the basis of the MTO and MTS mixed production mode of iron and steel enterprises, this paper will make order promising based on short-term production capacity balancing planning in line with the actual conditions of iron and steel enterprises. RATP considers that overall resource promising is a kind of order quantity promising. Furthermore, CTP means spare production capacity of the 
equipment promising after finishing the production plan, its calculation focuses on utilizing the equipment spare production capacity to satisfy new order capacity demands, while the original load of equipment is unchanged. In this paper CTP involves delivery commitment, thus we call it DTP. DTP, which is an extension of RATP, is delivery promising which aims at responding to customer's delivery requests more accurately. Because MTO part is a pre-signed order with resources that have been pre-occupied, the commitment of resources for new orders only contains two parts in MTS and surplus production capacity of resources. These three parts of resources are shown as given below.

1) Entirely matching resources. They include resources which have the same variety, specification and process with the inquiry orders, resources of MTS parts (including stock and virtual scheduling) which have not matched yet.

2) Alternative resources. They refer to MTS products which have not been matched with orders. Alternative resources can be divided into two categories: one is the resource with similar variety, specification and the same process routes, processing time with customer's orders. Another kind of resources has different varieties, specifications, but the same process route, processing time.

3) Resources of surplus production capacity. Surplus production capacities are the production capacities, not being occupied by the MTO and MTS parts. They are also available resources in the ordering promising.

Order promising in a steel enterprise is finding resources which could meet order conditions from entirely matching resources, alternative resources and resources of surplus production capacity to delivery orders, based on customer orders demands in accordance with the metallurgic products' specifications, quality and technology standards. The specific matching process is described briefly in Fig. 1.

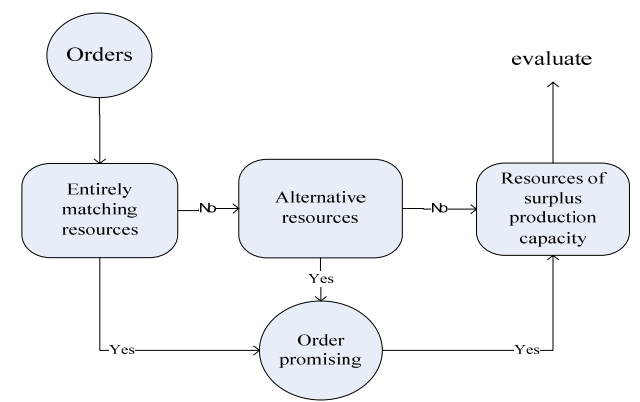

Fig. 1. Order promising process

As shown in Fig. 1, ATP in entirely matching resources needs to be checked firstly, if this part of resources could not fully satisfy the customer's order and then check ATP in alternative resources to fulfil it, meanwhile calculating the cost and production capacity adjustment. If it is still unsatisfied, the resources of surplus production capacity are checked and the production cost is calculated. For orders which could not be satisfied by the above three resources, their importance should be evaluated before deciding whether a former production plan should be adjusted. If it is requested to be adjusted, the production capacity is calculated while orders are executed. 
Aiming at responding quickly to customers, speeding up the turnover of corporate liquidity and reducing the cost of enterprise resource, the goals of resource promising are: (1) Punctuality of delivery. Providing customers with products in time is not only an important criterion to evaluate corporate reputation, but also a crucial means to win the trust of customers. (2) Minimize the resource promising cost. There are different costs raised by the resource replacements between the steel grades, which require that the cost must be lower than the sales price. In addition, during the promising by alternative resources, some processing cost is necessary to fulfil the order requirement. (3) Maximize the quantity of resource promising. The purpose of order promising is making appropriate orders to promise as much as possible, so that the order quantity is achieved.

Based on the above objectives, the constraints thought over for order promising in this paper are: 1) Delivery date. Companies should be able to deliver within the delivery date, otherwise give the expected delivery time in order to negotiate with customers. 2) Production process and production capacity. Companies should consider the overall capacity balance between the processes and process constraints through resources available-to-promise in order promising. 3) Manufacturing cost. Execute adjustment only if the cost is less than the profits of order product.

\subsection{Model formulation}

To simplify the modelling, we assume that each order has a single product variety and specification, and meanwhile it can employ multiple resources to make promising. Taking the steelmaking to a hot rolling production process for example, the following notations are introduced:

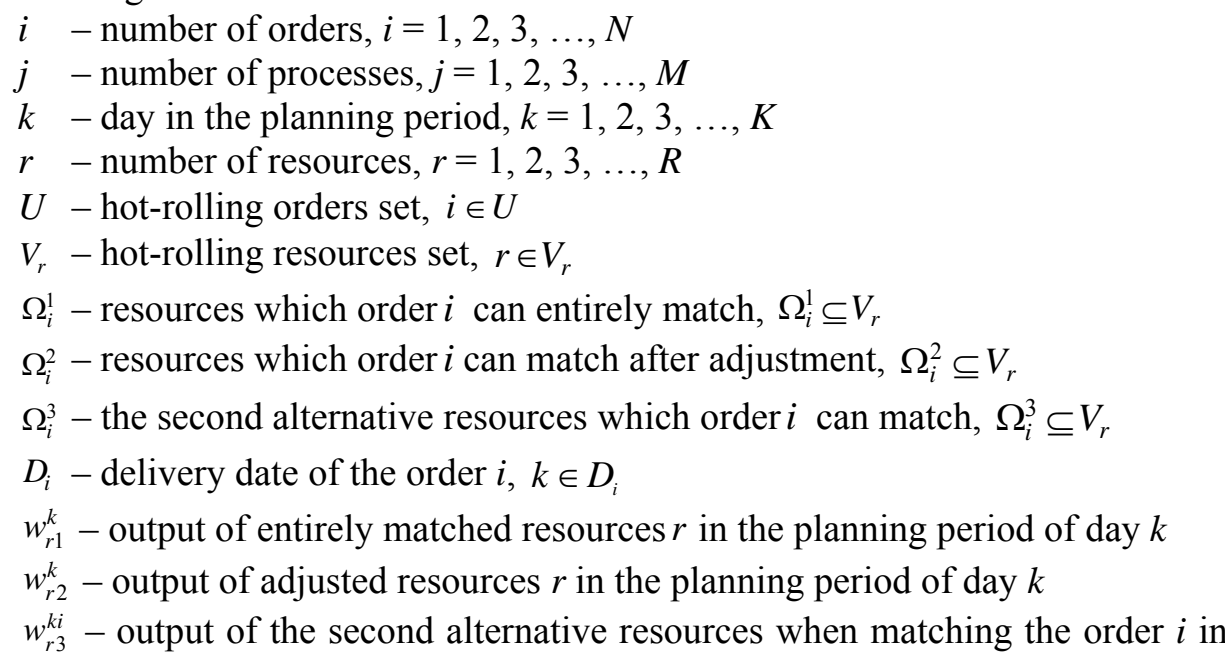
the planning period of day $k$

$w_{r 3}^{j k}$ - output of the second alternative resources in the process $j$ in period $k$

$\omega_{r 3}^{j}$ - production capacity of the second alternative resources $r$ needed in process $j$ (Unit/ tons per 1 hour) per 1 unit

$\omega_{i}^{j}$ - production capacity order $i$ needed in process $j$ per unit 
$E_{j}$ - surplus production capacity of process $j$ in the current planning period (Unit/ hours)

$c_{r}^{2}$ - adjustment costs of the adjusted matching resources $r$ per unit

$c_{r}^{4 j}$ - production cost of the surplus production capacity resources in process $j$

of order $i$ per unit

$O_{i}$ - ordering quantity of order $i$

$b_{i}^{l}$ - production lot size of order $i$.

The decision variables are as follows:

$x_{i r}^{1}=\left\{\begin{array}{l}1 \text { match to order } i \text { by entirely matching resources } r, \\ 0 \text { otherwise; }\end{array}\right.$

$y_{i r}^{2}=\left\{\begin{array}{l}1 \text { match to order } i \text { by alternative matching resources } r, \\ 0 \text { otherwise; }\end{array}\right.$

$z_{i j}^{3}=\left\{\begin{array}{l}1 \text { match to order } i \text { by the second alternative resources } r, \\ 0 \text { otherwise; }\end{array}\right.$

$u_{i r}^{4}=\left\{\begin{array}{l}1 \text { match to order } i \text { bysurplus production capacity resource } r, \\ 0 \text { otherwise. }\end{array}\right.$

Now we can construct a model of resource promising as follows:

Objective function

$$
\begin{gathered}
\max f_{1}=\sum_{i \in U} \sum_{r \in \Omega_{i}^{j}} \sum_{k=0}^{D_{i}} x_{i r}^{1} w_{r 1}^{k}+\sum_{i \in U} \sum_{r \in \Omega_{i}^{2}} \sum_{k=0}^{D_{i}} y_{i r}^{2} w_{r 2}^{k}+\sum_{i \in U} \sum_{r \in \Omega_{i}^{3}} \sum_{k=0}^{D_{i}} z_{i j}^{3} w_{r 3}^{k i} \omega_{r 3}^{j} / \omega_{i}^{j}+ \\
+\sum_{i \in U} u_{i r}^{4} E_{j} \omega_{i}^{j}, \\
\min f_{2}=\sum_{i \in U} \sum_{r \in \Omega_{i}^{2}} \sum_{k=0}^{D_{i}} y_{i i}^{2} c_{r}^{2} w_{r 2}^{k}+\sum_{i \in U} \sum_{r \in \Omega_{i}^{3}} \sum_{j=0}^{M} \sum_{k=0}^{D_{i}} z_{i j}^{3} c_{r}^{4 j} w_{r 3}^{k i} \omega_{r 3}^{j} / \omega_{i}^{j}+\sum_{i \in U} u_{i r}^{4} \sum_{j=0}^{M} c_{r}^{4 j} E_{j} \omega_{i}^{j}
\end{gathered}
$$

subject to:

$$
\begin{aligned}
\sum_{r \in \Omega_{i}^{i}} \sum_{k=0}^{D_{i}} x_{i r}^{1} w_{r 1}^{k}+\sum_{r \in \Omega_{i}^{2}} \sum_{k=0}^{D_{i}} y_{i r}^{2} w_{r 2}^{k}+\sum_{r \in \Omega_{i}^{3}} \sum_{k=0}^{D_{i}} z_{i r}^{3} w_{r 3}^{k i} \omega_{r 3}^{j} / \omega_{i}^{j}+u_{i r}^{4} E_{j} \omega_{i}^{j} \leq O_{i}, i \in U, \\
\left\{\begin{array}{l}
w_{r 3}^{k i}=w_{r 3}^{j k} \omega_{r 3}^{j} / \omega_{i}^{j} \text { if } w_{r 3}^{j k} \omega_{r 3}^{j} / \omega_{i}^{j} \geq b_{i}^{l}, \\
w_{r 3}^{k i}=0 \text { otherwise, }
\end{array}\right.
\end{aligned}
$$

$$
E_{j} \omega_{i}^{j} \geq b_{i}^{l} .
$$

In this model function (1) means maximizing the quantity of resource promising, including entirely matching resources, adjusted matching resources, the second alternative resources and surplus production capacity resources. The objective (2) denotes minimizing the cost of resource promising. Constraint (3) ensures that the quantity of actual resources promising should not exceed the order quantity. Constraint (4) shows the minimum production lot size when the second alternative resources match to order $i$, it should not be matched if violated. Constraint (5) states that the output of order $i$ should not be less than the minimum production lot size when using surplus production capacity resources to promise order. 


\section{Order promising algorithm based on resource balancing planning}

Although the model defined in Section 2.2 can give optimization of the order commitment, timely commitment is necessary because the customers' demands arrive one after another. We have developed a quick order promising method on the basis of optimization of the short-term production capacity balancing planning model [10]. By this method, order promising is not the traditional inventory matching, but a kind of promising to all the enterprise resources, which is called RATP, meanwhile, DTP is also promised.

For the sake of realizing the goals of the model in Section 2.2, the overall resources should first be balanced and optimized before order promising. Then rebalance and re-optimize the overall production given a changeable resource and promising condition. The whole order promising process based on resource balancing planning is as follows:

Step 1. Generate capacity balancing scheduling. Dynamic calculating MTO resources of each order once in turn and then processing order promising based on capacity balance, besides order quantity promising and delivery promising are processed simultaneously.

Step 2. RATP and DTP promising for new customer inquiring.

Step 3. In line with the current order promising and resources change, return to Step1 to re-optimize.

The overall resource balancing is achieved using the short-term production capacity balancing planning model we introduced in an essay [10], because the order promising model which dynamically calculates the executing MTS resources, mainly deals with solutions of short-term production capacity balancing planning. Hence, the primary work in the order promising stage is promising the customer quickly and giving suitable promising results. In this problem, the orders we need to cross-calculate include customer's orders, unexecuted orders and new orders and meanwhile the process for orders is dynamic, thus traditional optimal algorithms are unsuitable. We employ a heuristic method for order promising because of the characteristics of the problem and the limitation of the optimal algorithms. The main process is as following:

Step 1. Inquiry form is accepted from the customer.

Step 2. Resources matching. The process using a heuristic algorithm is stated as follows:

a) Match entirely matching resources. Count the unmatched production before the delivery date and check whether the quantity meets the demand of the inquiry form. Go to Step 4 if it does. Otherwise, calculate the satisfied quantity, tardiness delivery quantity, and employ alternative resources to match for the unfinished part.

b) Match the alternative resources. Select the resource for which the adjustment costs are less than the income, then match the resource with the minimal one successively. Count the unmatched production before the delivery date and check whether the quantity meets the demand of inquiry form. Obtain order promising and go to Step 4 if the demand is satisfied. Otherwise, calculate the 
unfinished quantity and use the second alternative resources to match.

c) Match the second alternative resources. Calculate if the inequality holds, then count the unmatched production before the delivery date and check whether the quantity meets the demand of the inquiry form. Give promising if satisfied and update the resource data. Otherwise, calculate the unfinished quantity and use resources of surplus production capacity to match.

d) Match resources of the surplus production capacity. According to the process constraint, calculate if the surplus production capacity meets the unfinished quantity of inquiry form before expected delivery date. Go to Step 4 if the unfinished quantity of the inquiry form is satisfied. Otherwise, calculate the promising quantity of all above three kinds of resources for the inquiry form.

Step 3. Assess the optimization results and make decisions. Assess whether the decision needs the whole resource optimization to meet the demand of the inquiry form. Give promising quantity if the whole resource optimization is not necessary, and then go to Step 4. Otherwise, start short-term production capacity balancing planning optimization algorithm with the goal of on time delivery, and go to Step 5 .

Step 4. Give the quantity and date promising.

Step 5. Order affirmation. Affirm the quantity and date of delivery with customers. Ensure that the promising quantity is less than the result of resources matching.

Step 6. Resources allocation. Allocate the resources to relevant orders according to the demands.

Consequently, the process of order promising of RATP and DTP based on short-term production capacity balancing plan is shown in Fig. 2.

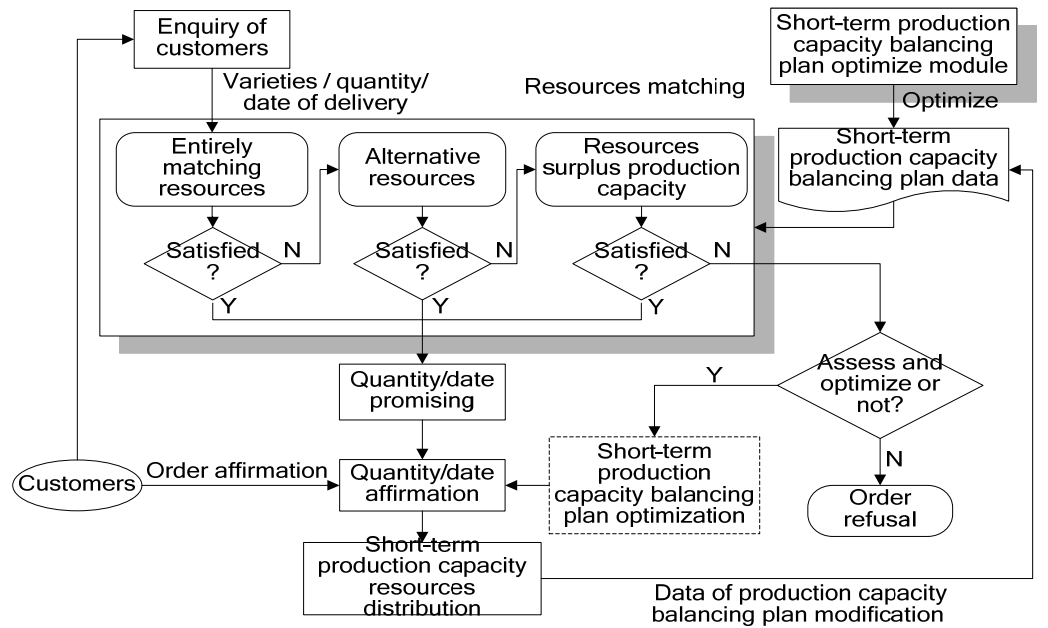

Fig. 2. Plot of the order promising process

\section{Experiment and results}

A case study of short-term balancing planning, including 200 order specifications has been conducted to evaluate the performance and validity of the proposed model. 
The equipment units contain 3 Basic Oxygen Furnace units (3, BOF), an Argon Blowing Station (AS), a CAS refining station, a LF finery, a RH refining station, 2 Continuous Casting Machines CCM1 and CCM2, a 2160 Continuous Casting Machine, a 1580 Hot Rolling Machine, a 2160 Hot Rolling Machine. The arrows in the figure show the production processes. Fig. 3 indicates that this is a complex production network of the manufacturing processes, an alternative process route among different products which impact each other, and products with the same variety and specification exist between two hot-rolling production lines. All the above problems increase the complexity of the order promising. The main production equipment and process network is shown in Fig. 3.

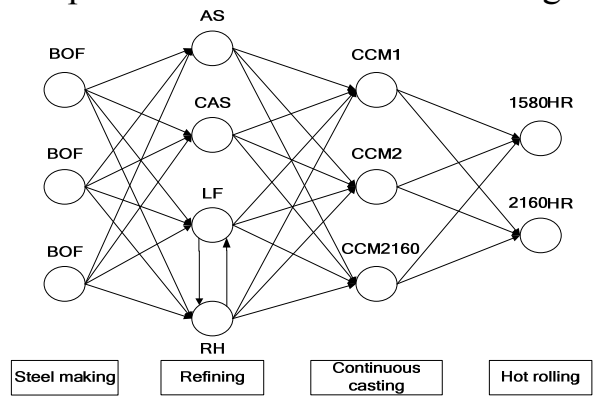

Fig. 3. Plot of the process production network

There are 20 sales orders with 5 varieties, 9 processes routes and 37656 total quantity demands needing rapid promising one day in the order stage. For simply representing the order inquiring sequence, we number the orders in a unified number. The order information is revealed as given in Table 1.

Table 1. Data of order information

\begin{tabular}{|c|c|c|c|c|c|c|}
\hline Order No & Steel grade & Width & Thickness & Processes & Quantity & Delivery date \\
\hline q9000001 & Q235B & 950 & 2.30 & 1368 & 1544 & 2012301 \\
\hline q9000002 & Q235B & 950 & 7.80 & 1368 & 1571 & 2012302 \\
\hline q9000003 & Q345C & 950 & 3.50 & 1268 & 1523 & 2012306 \\
\hline q9000004 & Q345C & 950 & 7.80 & 1479 & 1886 & 2012302 \\
\hline q9000005 & Q235B & 950 & 10.20 & 1368 & 1574 & 2012302 \\
\hline q9000006 & Q235B & 1100 & 2.30 & 1369 & 2269 & 2012303 \\
\hline q9000007 & Q235B & 1100 & 2.56 & 1369 & 1789 & 2012302 \\
\hline q9000008 & Q235B & 1100 & 10.20 & 1468 & 1689 & 2012303 \\
\hline q9000009 & Q235B & 1220 & 2.30 & 1468 & 1694 & 2012302 \\
\hline q90000010 & Q235C & 1400 & 7.80 & 1469 & 1747 & 2012304 \\
\hline q90000011 & Q235C & 1400 & 10.20 & 1469 & 1584 & 2012304 \\
\hline q90000012 & Q235C & 1600 & 2.30 & 1579 & 1941 & 2012303 \\
\hline q90000013 & Q235D & 1220 & 7.80 & 1379 & 1943 & 2012306 \\
\hline q90000014 & Q345B & 1800 & 10.20 & 1579 & 2372 & 2012302 \\
\hline q90000015 & Q345B & 1900 & 2.30 & 1579 & 2463 & 2012301 \\
\hline q90000016 & Q345B & 1900 & 10.20 & 1579 & 2011 & 2012303 \\
\hline q90000017 & Q345C & 950 & 2.56 & 1468 & 1717 & 2012306 \\
\hline q90000018 & Q345C & 950 & 10.20 & 1479 & 2437 & 2012303 \\
\hline q90000019 & Q235B & 1220 & 10.20 & 1568 & 2304 & 2012306 \\
\hline q90000020 & Q235C & 1800 & 2.30 & 1569 & 1598 & 2012303 \\
\hline Total & & & & & 37656 & \\
\hline
\end{tabular}


We defined the promising type, each of which indicates the resources type for which orders are matched: 0 - No matching resources; $1 \sim$ - entirely matching resources; 2.1 - alternative resources of the same series and same process; $2.2 \tilde{-}$ alternative resources of different series and the same process; $2.3 \tilde{-}$ alternative resources of different series and different process; 3 - resources of surplus production capacity. By the order promising method, we inquired the order shown in Table 1 one by one. Table 2 displays the order promising results.

Table 2. Data of order promising results

\begin{tabular}{|c|c|c|c|c|c|}
\hline Order No & $\begin{array}{l}\text { Specification } \\
(\mathrm{mm} \times \mathrm{mm})\end{array}$ & $\begin{array}{l}\text { Product variety } \\
\text { for promising } \\
\text { processes }\end{array}$ & $\begin{array}{l}\text { Promising } \\
\text { quantity }(\mathrm{t})\end{array}$ & $\begin{array}{l}\text { Delivery } \\
\text { date }\end{array}$ & $\begin{array}{l}\text { Promising } \\
\text { type }\end{array}$ \\
\hline q9000001 & $950 \times 2.30$ & Q235B - 1368 & 1544 & 2012301 & 1 \\
\hline q9000002 & $950 \times 7.80$ & Q235B - 1368 & 1571 & 2012302 & 1 \\
\hline q9000003 & $950 \times 3.50$ & Q345C - 1268 & 1523 & 2012306 & 1 \\
\hline q9000004 & $950 \times 7.80$ & Q345C - 1479 & 1474 & 2012304 & 3 \\
\hline q9000005 & $950 \times 10.20$ & Q345B - 1268 & 1218 & 2012305 & 2.3 \\
\hline q9000006 & $1100 \times 2.30$ & $\mathrm{Q} 235 \mathrm{C}-1369$ & 1984 & 2012303 & 2.1 \\
\hline q9000007 & $1100 \times 2.56$ & Q235B - 1369 & 1789 & 2012303 & 1 \\
\hline$q 9000008$ & $1100 \times 10.20$ & Q235B-1468 & 1468 & 2012303 & 1 \\
\hline q9000009 & $1220 \times 2.30$ & Q235B - 1468 & 1694 & 2012303 & 1 \\
\hline q90000010 & $1400 \times 7.80$ & $\mathrm{Q} 235 \mathrm{C}-1469$ & 1590 & 2012305 & 1 \\
\hline q90000011 & $1400 \times 10.20$ & Q235C - 1469 & 1476 & 2012305 & 1 \\
\hline q90000012 & $1600 \times 2.30$ & Q345D - 1579 & 1635 & 2012305 & 2.2 \\
\hline q90000013 & $1220 \times 7.80$ & Q235B-1579 & 1943 & 2012302 & 2.2 \\
\hline q90000014 & $1800 \times 10.20$ & Q235B - 1579 & 1821 & 2012302 & 2.2 \\
\hline \multirow{2}{*}{ q90000015 } & \multirow{2}{*}{$1900 \times 2.30$} & Q345B - 1269 & 1789 & 2012305 & 1 \\
\hline & & Q345C - 1279 & 674 & 2012306 & 2.1 \\
\hline q90000016 & $1900 \times 10.20$ & Q345B - 1279 & 1531 & 2012305 & 1 \\
\hline q90000017 & $950 \times 2.56$ & Q345D - 1468 & 1717 & 2012306 & 2.1 \\
\hline q90000018 & $950 \times 10.20$ & Q235D - 1368 & 1894 & 2012303 & 1 \\
\hline q90000019 & $1220 \times 10.20$ & Null & 0 & 0 & 0 \\
\hline q90000020 & $1800 \times 2.30$ & Null & 0 & 0 & 0 \\
\hline $\begin{array}{l}\text { Total } \\
\text { weight }\end{array}$ & & & 30335 & & \\
\hline
\end{tabular}

The orders with tail numbers $1,2,3,7,8,9,10,11,16,18$ are matched with entirely matching resources with the same variety, specification and process, while orders with a tail number 6 or 17 are matched with alternative resources which have the same specification, but different processes. The order q90000015 is matched with entirely matching resources and alternative resource. Orders with tail numbers $12,13,14$ are totally promised by the alternative resources of different series, but same process and order $\mathrm{q} 9000005$ is promised by the surplus production capacity. Since there are no suitable resources for order requirement, order q90000019 and q90000020 are matched by other ways. The statistical result of promising based on resource balancing planning is stated in Table 3 according to the current resource allocation and utilization conditions.

Besides, for verifying the feasibility and efficiency of the proposed method and algorithm, we compared proposed method A with the traditional ATP methods. Method A is a traditional ATP method using the resource balance strategy, which 
uniquely adopts stock matching and surplus spare capacity to satisfy the delivery demand and customer commitment. Method B is a traditional method in which the resource balancing planning strategy is not ignored. Method $\mathrm{C}$ is the proposed method in which the alternative resources, surplus production capacity and entirely matching resources are taken into account. The computational results are stated in Table 3. In Table 3 the Total Promising Quantity rate - TPQ, Order Promising rate - OP, Promising Quantity Satisfy rate- PQS, Order Promising Quantity Satisfy rate - OPQS, Delivery Data Satisfied rate - DDS are given.

Table 3. Statistical result of promising

\begin{tabular}{|l|c|l|c|}
\hline \multicolumn{1}{|c|}{ Item } & Statistical result & \multicolumn{1}{|c|}{ Item } & Statistical result \\
\hline Number of the inquiry form & 18 & Quantity of promising by mode 2.3 & 1218 \\
\hline Quantity of promising (tons) & 30335 & Quantity of promising by mode 3 & 1474 \\
\hline $\begin{array}{l}\text { Quantity of promising by } \\
\text { mode 1 }\end{array}$ & 17869 & Quantity of promising by a hybrid mode & 2463 \\
\hline $\begin{array}{l}\text { Quantity of promising by } \\
\text { mode 2.1 }\end{array}$ & 4375 & Quantity of partly promising & 412 \\
\hline $\begin{array}{l}\text { Quantity of promising by } \\
\text { mode 2.2 }\end{array}$ & 5399 & Quantity of completely not promising & 3902 \\
\hline
\end{tabular}

The promising algorithm is based on the resource balancing plan, and the enquiry time for each order is less than $1.0 \mathrm{~s}$. Meanwhile, it can ensure the balance of the overall production capacity and logistics of the enterprise. In Table 3 we can see that the percentage of promising by mode 1 is $58.9 \%$ which means that orders are mostly matched by mode 1 which is entirely matching resources. Moreover, the percentage of promising by modes $2.1,2.2$ and 2.3 is $36.2 \%$, thus reminding us that alternative resources could be useful in order promising in this case. Additionally, there are $4 \%$ resources of surplus production capacity being utilized in a matching orders which is higher than the quantity of promising by mode 2.3 and meanwhile the percentage of promising by a hybrid mode is $8 \%$ which is not only higher than mode 2.3 , but also not less than mode 3 , so hybrid mode promising could be meaningful in order promising based on the production capacity balance. Finally, the percentage of quantity of completely not promising is $10 \%$. The test shows that order promising based on the production capacity balancing planning involved in rule-based searching, matching and adjustment to achieve promising to customers, is feasible and helpful in controlling the inventory from the root cause as much as possible.

Besides, for verifying the feasibility and efficiency of the proposed method and algorithm, we compared the proposed method A with the traditional ATP methods. Method A is a traditional ATP method using the resource balance strategy, which uniquely adopts stock matching and surplus spare capacity to satisfy the delivery demand and customer commitment. Method B is a traditional method in which the resource balancing planning strategy is not ignored. Method $\mathrm{C}$ is the proposed method in which the alternative resources, surplus production capacity and entirely matching resources are taken into account. The computational results are stated in Table 3. In Table 3, TPQ, OP, PQS, OPQS, DDS are shown. 
Table 4. Methods comparison results and analysis

\begin{tabular}{|l|l|l|l|l|l|}
\hline Type & TPQ & OP & PQS & OPQS & DDS \\
\hline Method A & $42.5 \%$ & $55 \%$ & $35 \%$ & $63.6 \%$ & $45.5 \%$ \\
\hline Method B & $29.1 \%$ & $35 \%$ & $5 \%$ & $14.2 \%$ & $42.9 \%$ \\
\hline Method C & $80.6 \%$ & $90 \%$ & $65 \%$ & $77.8 \%$ & $50 \%$ \\
\hline
\end{tabular}

Table 4 reveals that Method $\mathrm{C}$ which is proposed, has better performance in TPQ, OP, PQS, OPQS and DDS than methods A and B, which indicates that the proposed RATP and DTP which considered the overall resources matching and date of delivery calculation has priority in these aspects. Moreover, we notice that method A has higher rates in the above indicators than method B; it expresses that adopting a resource balancing planning strategy is feasible and efficient.

In our testing experiment, the processing time for each enquiry order is less than $1.0 \mathrm{~s}$ and it can ensure the balance of the overall production capacity and logistics of the enterprise. The testing experiment shows that this method can meet the customer's order promising in reality. The result shows that the order promising based on production capacity balancing planning using rule-based searching, matching and adjustment to achieve promising to customers, is efficient; during which the transform capacity occupied by planned orders in advance of the demands of new orders utilizes the capacity replacement method. Trying to control the inventory from the root cause as much as possible on the basis of the production capacity balance is possible and dynamical adjustment of the production plans in order to ensure smooth logistics and production balance of the enterprise as a whole can be ensured by order promising based on production capacity balancing planning.

\section{Conclusion}

On the basis of production and sales resources balancing planning, the concepts of RATP and DTP were proposed, which means overall resources matching and date of delivery calculation. This method can efficiently guarantee the balanced utilization of enterprises' overall production resources by short-term capacity balancing planning optimization. On the foundation of the overall balancing results, quantity and date of delivery can be promised in time by a heuristic RATP and DTP method. Furthermore, production capacity of orders has been formed and orders which have not been executed with new orders are rolling calculated. The implementation of the dynamic process for orders reduces the possibility of stock, which consequently ensures smooth logistics and balance of the enterprises' overall production and sales resources.

Acknowledgements: This work was supported by the National High-tech R\&D Program of China under Grant No 2013AA040704 and National Natural Science Foundation of China under Grant No 51274043. 


\section{References}

1. B u i, T., H. J. S e b a sti a n. Integration of Multi-Criteria Decision Analysis and Negotiation in Order Promising. - In: Proc. of 43rd HICCS Conference, Honolulu, 2010, pp. 1-10.

2. Chen, C., M. Siddharth, D. Purushothaman, B. Wang. The Capacity Planning Problem in Make-to-Order Enterprises. - Mathematical and Computer Modelling, Vol. 50, No 9-10, 2009, pp. 1461-1473.

3. Che n, J.-H., C. Chen. Using Mathematical Programming on Two-Phase Order Promising Process with Optimized Available-to-Promise Allocation Planning. - International Journal of the Computer, the Internet and Management, Vol. 17, No 3, 2009, pp. 25-40.

4. Christos, T., T. Sung. Integration of Production Planning and Scheduling: Overview, Challenges and Opportunities. - Computers \& Chemical Engineering, Vol. 33, 2009, No 12, pp. 1919-1930.

5. He m p s c h, C., H. J. S e b a s t i a n, T. B u i. Solving the Order Promising Impasse Using MultiCriteria Decision Analysis and Negotiation. - Logistics Research, Vol. 6, 2013, No 1, pp. 25-41.

6. Jung, H. S. An Available-to-Promise Model Considering Customer Priority and Variance of Penalty Costs. - International Journal of Advanced Manufacturing Technology, Vol. 49, 2010, No 1-4, pp. 369-377.

7. L u, S. Supply Chain-Oriented Multi-Stage ATP Study. - Intelligent Computation Technology and Automation (ICICTA). Hunan, 2008, pp. 712-716.

8. M e y r, H. Customer Segmentation, Allocation Planning and Order Promising in Make-to-Stock Production. - Supply Chain Planning, Vol. 31, 2009, pp. 229-256.

9. Mo 1 i n è, J. I., A. M. C o ve s. Order Allocation in a Multi-Supplier Environment: Review of the Literature Since 2007. - Journal of Industrial Engineering and Management, Vol. 6, 2012, No 3, pp. 139-146.

10. S o n g, X., Z. L v, S. D o n g. Network Capacity-Based Algorithm for Short Term Production Capacity Planning Problem in Steel Plant. - Computer Integrated Manufacturing System, Vol. 18, 2012, No 3, pp. 1839-1844.

11. T s a i, K. M., Y. J. C h e n, S. Y. Y o u. Channels Focused Production and Allocation Planning for a Steel Supply Chain. - A Fuzzy Goal Programming with Priority Approach. - Foundations of Computing and Decision Sciences, Vol. 34, 2009, No 2, pp. 125-152.

12. Z h a n g, J., B. H a n, L. N i e. Research on Capacity Calculation and Assessment Framework for Chinese High Speed Railway Based on UIC406. - Journal of System and Management Sciences, Vol. 1, 2011, No 6, pp. 59-75.

13. Z h a n g, T., H. Ch en g, Y. Z h a n g. A Method Based on MTO-MTS for the Order Planning of the Steel Plant. - Systems Engineering-Theory \& Practice, Vol. 11, 2008, pp. 85-89. 